\title{
Generalized convolution properties based on the modified Mittag-Leffler function
}

\author{
H. M. Srivastavaa ${ }^{a, b}$, Adem Kılıçmann, ${ }^{c, *}$, Zainab E. Abdulnaby, ${ }^{c, d}$, Rabha W. Ibrahim ${ }^{e}$ \\ ${ }^{a}$ Department of Mathematics and Statistics, University of Victoria, Victoria, British Columbia V8W 3R4, Canada. \\ ${ }^{b}$ Center for General Education (Department of Science and Technology), China Medical University, Taichung 40402, Taiwan, Republic \\ of China. \\ ${ }^{c}$ Department of Mathematics, Universiti Putra Malaysia, 43400 UPM Serdang, Selangor, Malaysia. \\ ${ }^{d}$ Department of Mathematics, College of Science, Al-Mustansiriyah University, Baghdad, Iraq. \\ ${ }^{e}$ Faculty of Computer Science and Information Technology, University of Malaya, 50603 Kuala Lumpur, Malaysia.
}

Communicated by A. Atangana

\begin{abstract}
Studies of convolution play an important role in Geometric Function Theory (GFT). Such studies attracted a large number of researchers in recent years. By making use of the Hadamard product (or convolution), several new and interesting subclasses of analytic and univalent functions have been introduced and investigated in the direction of well-known concepts such as the subordination and superordination inequalities, integral mean and partial sums, and so on. In this article, we apply the Hadamard product (or convolution) by utilizing some special functions. Our contribution in this paper includes defining a new linear operator in the form of the generalized Mittag-Leffler function in terms of the extensively-investigated Fox-Wright ${ }_{p} \Psi_{q-}$ function in the right-half of the open unit disk where where $\mathfrak{R}(z)>0$. We then show that the new linear convolution operator is bounded in some spaces. In particular, several boundedness properties of this linear convolution operator under mappings from a weighted Bloch space into a weighted-log Bloch space are also investigated. For uniformity and convenience, the Fox-Wright ${ }_{\mathrm{p}} \Psi_{\mathrm{q}}$-notation is used in our results. (C)2017 All rights reserved.
\end{abstract}

Keywords: Fractional calculus, analytic functions, fractional calculus operator, univalent functions, convex functions, Mittag-Leffler function, Fox-Wright $p \Psi_{q}$-function, weighted $\mu$-Bloch space, weighted-log Bloch space.

2010 MSC: 33E12, 26A33, 47B38.

\section{Introduction}

Various operators provide tools of considerable significance in the subject of the functional analysis and they are known to have applications in numerous additional areas of pure and applied mathematics. It is well-known that these various types of operators, such as linear operators, differential and integral operators and fractional operators. The studies of operators appear in many fields; for instance, in classical mechanics, the derivative operator is utilized ubiquitously, and (in quantum mechanics) observation

\footnotetext{
*Corresponding author

Email addresses: harimsri@math.uvic.ca (H. M. Srivastava), akilic@upm.edu.my (Adem K1lıçman), esazainab@yahoo.com (Zainab E. Abdulnaby), rabhaibrahim@yahoo.com (Rabha W. Ibrahim)

doi:10.22436/jnsa.010.08.23
}

Received 2017-02-24 
is signified by Hermitian operators. Essential properties that many operators may display include boundedness, linearity and continuity. The area of Geometric Function Theory (GFT) is remarkably very rich in various classes of interesting operators. These operators are classified due to their operational mechanism. Essentially, these operators are used to define, improve, and generalize many well-known analytic function classes in the open unit disk. These studies imply more geometric features for the investigated analytic function classes and preserve many of their properties (see, for example, [1, 2, 10, 26, 27]).

In this study, we introduce a new linear operator in the open unit disk by making use of the convolution product (or the Hadamard product) of some special functions (such as the Mittag-Leffler function and the Fox-Wright ${ }_{p} \Psi_{q}$-function). Our studies are based on various boundedness properties and illustrate a number of other geometric properties of the convolution operator which is introduced here. The generalized Mittag-Leffler function plays a significant role in the theory of fractional calculus with its applications in engineering and physics due to their vast potential and asymptotic properties (see, for example, [26, 27]; see also [5, 6] as well as the references which are cited in each of these recent works).

\section{Preliminaries}

In this section, we recall some basic definitions and concepts.

Let $\mathcal{H}(\mathbb{U})$ denote the class of functions which are analytic in the open unit disk

$$
\mathbb{U}:=\{z: z \in \mathbb{C} \text { and }|z|<1\} .
$$

For $k \in \mathbb{N}=\{1,2,3, \cdots\}$ and $a \in \mathbb{C}$, let

$$
\mathcal{H}[\mathrm{a}, \mathrm{k}]=\left\{\mathrm{f} \in \mathcal{H}(\mathbb{U}): \mathrm{f}(z)=\mathrm{a}+\mathrm{a}_{\mathrm{k}} z^{\mathrm{k}}+\mathrm{a}_{\mathrm{k}+1} z^{\mathrm{k}+1}+\cdots\right\}
$$

and $\mathcal{H}_{0}=\mathcal{H}[0,1]$. We denote by $\mathcal{A}$ the class of all functions of the form:

$$
f(z)=z+\sum_{k=2}^{\infty} a_{k} z^{k},
$$

which are analytic in $\mathbb{U}$. We denote by $\mathcal{S}$ and $\mathcal{K}$ the classes of all functions $f \in \mathcal{A}$ that are, respectively, univalent and convex in $\mathbb{U}$ and normalized by

$$
f(0)=f^{\prime}(0)-1=0 .
$$

Theorem 2.1 (Bieberbach Conjecture; de Branges' Theorem [4]). If the function $\mathrm{f}(z)$ defined by (2.1) is in the class $\mathcal{S}$, then

$$
\left|a_{k}\right| \leqq k \quad(k \in \mathbb{N} \backslash\{1\}) .
$$

Moreover, if the function $\mathrm{f}(\boldsymbol{z})$ defined by (2.1) is in the class $\mathcal{K}$, then

$$
\left|a_{k}\right| \leqq 1 \quad(k \in \mathbb{N} \backslash\{1\}) .
$$

Definition 2.2. Let the function $f(z)$ be given by (2.1) and let

$$
g(z)=z+\sum_{k=2}^{\infty} b_{k} z^{k} .
$$

Then the convolution (or Hadamard product) $(f * g)(z)$ of the two functions $f(z)$ and $g(z)$ is defined by

$$
(f * g)(z)=z+\sum_{k=2}^{\infty} a_{k} b_{k} z^{k}=(g * f)(z) .
$$

We now proceed to introduce some special functions related to the celebrated Mittag-Leffler function. 
Definition 2.3 (see $[3,7,28]$ ). The generalized (two parameters) Mittag-Leffler type function $E_{\alpha, \beta}(z)$ is defined by

$$
\mathrm{E}_{\alpha, \beta}(z)=\sum_{k=0}^{\infty} \frac{z^{\mathrm{k}}}{\Gamma(\alpha \mathrm{k}+\beta)}
$$

so that, obviously,

$$
\mathrm{E}_{\alpha, 1}(z)=\mathrm{E}_{\alpha}(z):=\sum_{\mathrm{k}=0}^{\infty} \frac{z^{\mathrm{k}}}{\Gamma(\alpha \mathrm{k}+1)} \quad \text { and } \quad \mathrm{E}_{1,1}(z)=e^{z},
$$

where $E_{\alpha}(z)$ is the celebrated Mittage-Leffler function (see [13]).

Further studies of the Mittag-Leffler type functions can be found in the recent works by Gorenflo et al. [5], Haubold et al. [6], Kilbas et al. [8, 9], Kiryakova [11], Li et al. [12], Saxena et al. [16, 17], Sharma et al. [18-20], Srivastava et al. [21, 23, 24, 26], and Tomovski et al. [27]. In particular, several interesting properties and characteristics of the Mittag-Leffer functions $E_{\alpha}(z)$ and $E_{\alpha, \beta}(z)$, as well as their known extensions and generalizations, were discussed by Saxena et al. [17], Gorenflo et al. [5], Srivastava et al. [25, 26], and Tomovski et al. [27].

Recently, Paneva-Konovska [14] studied the behaviour of some special functions $\widetilde{\mathrm{E}}_{\alpha, n}, \widetilde{\mathrm{E}}_{n, \beta}$, and $\widetilde{\mathrm{E}}_{n}$, which are related to the Mittag-Leffler functions in the complex $z$-plane $\mathbb{C}$ as follows:

$$
\begin{aligned}
& \widetilde{\mathrm{E}}_{\alpha, \mathrm{n}}(z):= \begin{cases}1, & (\mathrm{n}=0), \\
\Gamma(\mathrm{n}) z^{\mathrm{n}} \mathrm{E}_{\alpha, \mathrm{n}}(z), & (\mathrm{n} \in \mathbb{N}),\end{cases} \\
& \widetilde{\mathrm{E}}_{\mathrm{n}, \beta}(z):= \begin{cases}\frac{1}{1-z}, & (\mathrm{n}=0), \\
\Gamma(\beta) z^{\mathrm{n}} E_{\mathrm{n}, \beta}(z), & (\mathrm{n} \in \mathbb{N}),\end{cases}
\end{aligned}
$$

and

$$
\widetilde{\mathrm{E}}_{\mathrm{n}}(z):= \begin{cases}\frac{1}{1-z}, & (\mathrm{n}=0), \\ z^{\mathrm{n}} \mathrm{E}_{\mathrm{n}}(z), & (\mathrm{n} \in \mathbb{N}) .\end{cases}
$$

Among other interesting results, the following asymptotic formulas for the large values of the indices $\alpha$ and $\beta$ in the definitions (2.3) and (2.4) were given for $z \in \mathbb{C}$ (see, for details, [14]):

$$
E_{\alpha, n}(z)=\frac{1}{\Gamma(n)}\left[1+\Theta_{\alpha, n}(z)\right], \quad E_{n, \beta}(z)=\frac{1}{\Gamma(\beta)}\left[1+\Theta_{n, \beta}(z)\right], \quad \text { and } \quad E_{n}(z)=1+\Theta_{n}(z),
$$

where $\Theta_{\alpha, n}, \Theta_{n, \beta}$, and $\Theta_{n}$ are given by

$$
\begin{aligned}
& \Theta_{\alpha, n}(z)=\Gamma(n) \sum_{k=1}^{\infty} \frac{z^{k}}{\Gamma(\alpha k+n)} \rightarrow 0, \quad(n \rightarrow \infty), \\
& \Theta_{n, \beta}(z)=\Gamma(\beta) \sum_{k=1}^{\infty} \frac{z^{k}}{\Gamma(n k+\beta)} \rightarrow 0, \quad(n \rightarrow \infty),
\end{aligned}
$$

and

$$
\Theta_{\mathrm{n}}(z)=\sum_{\mathrm{k}=1}^{\infty} \frac{z^{\mathrm{k}}}{\Gamma(\mathrm{nk}+1)} \rightarrow 0, \quad(\mathrm{n} \rightarrow \infty),
$$

respectively. Here the (real or complex) parameters $\alpha$ and $\beta$ as well as the argument $z \in \mathbb{C}$ and fixed, $n \in \mathbb{N}$, and the power series in (2.8), (2.9), and (2.10) define holomorphic functions for $z \in \mathbb{C}$. Moreover, the convergence is uniform on any compact subset of $\mathbb{C}$. 
In our present sequel to [14], we propose to investigate various new families of linear operators which are based upon the above functions convoluting with the function $f(z) \in \mathcal{A}$ given by (2.1). In order to present our results in a notationally convenient manner, we need the function of the Fox-Wright Function ${ }_{p} \Psi_{q}\left(p, q \in \mathbb{N}_{0}\right)$ or ${ }_{p} \Psi_{q}^{*}\left(p, q \in \mathbb{N}_{0}\right)$ which is a generalization of the familiar generalized hypergeometric function ${ }_{p} F_{q}\left(p, q \in \mathbb{N}_{0}\right)$ with $p$ numerator parameters $a_{1}, \cdots, a_{p}$ and $q$ denominator parameters $b_{1}, \cdots, b_{q}$ such that

$$
a_{i} \in \mathbb{C}(i=1, \cdots, p) \text { and } \quad b_{j} \in \mathbb{C} \backslash \mathbb{Z}_{0}^{-}(j=1, \cdots, q),
$$

defined by (see, for details, [9, p. 56])

$$
\begin{aligned}
& p \Psi_{q}^{*}\left[\begin{array}{c}
\left(a_{1}, A_{1}\right), \cdots,\left(a_{p}, A_{p}\right) ; z \\
\left(b_{1}, B_{1}\right), \cdots,\left(b_{q}, B_{q}\right) ;
\end{array}\right] \\
& :=\sum_{k=0}^{\infty} \frac{\left(a_{1}\right)_{A_{1} k} \cdots\left(a_{p}\right)_{A_{p} k}}{\left(b_{1}\right)_{B_{1} k} \cdots\left(b_{q}\right)_{B_{q} k}} \frac{z^{k}}{k !}=\frac{\Gamma\left(b_{1}\right) \cdots \Gamma\left(b_{q}\right)}{\Gamma\left(a_{1}\right) \cdots \Gamma\left(a_{q}\right)} p \Psi_{q}\left[\begin{array}{c}
\left(a_{1}, A_{1}\right), \cdots,\left(a_{p}, A_{p}\right) ; \\
\left(b_{1}, B_{1}\right), \cdots,\left(b_{q}, B_{q}\right) ;
\end{array}\right], \\
& \left(\begin{array}{c}
\left.A_{i}>0 \quad(i=1, \cdots, p) ; \quad B_{j}>0 \quad(j=1, \cdots, q) ; 1+\sum_{j=1}^{q} B_{j}-\sum_{i=1}^{p} A_{i} \geqq 0\right),
\end{array}\right.
\end{aligned}
$$

where the equality in the convergence condition holds true for suitably bounded values of $|z|$ given by

$$
|z|<\nabla:=\left(\prod_{i=1}^{p} A_{i}^{-A_{i}}\right) \cdot\left(\prod_{j=1}^{q} B_{j}^{B_{j}}\right) .
$$

In the particular case when

$$
A_{i}=B_{j}=1 \quad(i=1, \cdots, p ; j=1, \cdots, q),
$$

we have the following relationship:

$$
{ }_{p} \Psi_{q}^{*}\left[\begin{array}{c}
\left(a_{1}, 1\right), \cdots,\left(a_{p}, 1\right) ; \\
\left(b_{1}, 1\right), \ldots,\left(b_{q}, 1\right) ;
\end{array}\right]={ }_{p} F_{q}\left[\begin{array}{c}
a_{1}, \cdots, a_{p} ; \\
b_{1}, \cdots, b_{q} ;
\end{array}\right]=\frac{\Gamma\left(b_{1}\right) \cdots \Gamma\left(b_{q}\right)}{\Gamma\left(a_{1}\right) \cdots \Gamma\left(a_{q}\right)} p \Psi_{q}\left[\begin{array}{c}
\left(a_{1}, 1\right), \ldots,\left(a_{p}, 1\right) ; \\
\left(b_{1}, 1\right), \ldots,\left(b_{q}, 1\right) ;
\end{array}\right],
$$

in terms of the generalized hypergeometric function ${ }_{p} F_{q}\left(p, q \in \mathbb{N}_{0}\right)$.

A natural further generalization and unification of the Hurwitz-Lerch Zeta function $\Phi(z, s, a)$ as well as the Fox-Wright function $p \Psi_{q}^{*}$ defined by (2.11) was accomplished recently by Srivastava et al. [24] by introducing essentially arbitrary numbers of numerator and denominator parameters. For recalling the definition of their multi-parameter extension, in addition to the symbol $\nabla$ defined by (2.12) with, of course,

$$
A_{i}=\rho_{i}(i=1, \cdots, p) \text { and } B_{j}=\sigma_{j}(j=1, \cdots, q) \text {, }
$$

that is,

$$
\nabla^{*}:=\left(\prod_{i=1}^{p} \alpha_{i}^{-\alpha_{i}}\right) \cdot\left(\prod_{j=1}^{q} \beta_{j}^{\beta_{j}}\right)
$$

the following notations will be employed:

$$
\Delta:=\sum_{j=1}^{\mathrm{q}} \sigma_{j}-\sum_{i=1}^{p} \rho_{i} \quad \text { and } \quad \Xi:=s+\sum_{j=1}^{q} \mu_{j}-\sum_{i=1}^{p} \lambda_{i}+\frac{p-q}{2} .
$$


The extended Hurwitz-Lerch Zeta function $\Phi_{\lambda_{1}, \cdots, \lambda_{p} ; \mu_{1}, \cdots, \mu_{q}}^{\left(\rho_{1}, \cdots, \rho_{p} ; \sigma_{1}, \cdots, \sigma_{q}\right)}(z, s, a)$ is then defined by

$$
\begin{aligned}
& \Phi_{\lambda_{1}, \cdots, \lambda_{p} ; \mu_{1}, \cdots, \mu_{q}}^{\left(\rho_{1}, \ldots, \sigma_{q}\right)}(z, s, a)=\sum_{k=0}^{\infty} \frac{\prod_{i=1}^{p}\left(\lambda_{i}\right)_{k \rho_{i}}}{k ! \prod_{j=1}^{q}\left(\mu_{j}\right)_{k \sigma_{j}}} \frac{z^{k}}{(k+a)^{s}}, \\
& \left(p, q \in \mathbb{N}_{0} ; \lambda_{i} \in \mathbb{C}(i=1, \cdots, p) ; \quad a, \mu_{j} \in \mathbb{C} \backslash \mathbb{Z}_{0}^{-}(j=1, \cdots, q) ;\right. \\
& \rho_{i}, \sigma_{j} \in \mathbb{R}^{+}(i=1, \cdots, p ; j=1, \cdots, q) ; \quad \Delta>-1 \text { when } s, z \in \mathbb{C} ; \\
& \left.\Delta=-1 \text { and } s \in \mathbb{C} \text { when }|z|<\nabla^{*} ; \quad \Delta=-1 \text { and } \mathfrak{R}(\Xi)>\frac{1}{2} \text { when }|z|=\nabla^{*}\right),
\end{aligned}
$$

where $\mathbb{Z}_{0}^{-}$denotes the set of non-positive integers and $(\lambda)_{v}$ denotes the general Pochhammer symbol defined, in terms of the familiar gamma function, by

$$
(\lambda)_{v}:=\frac{\Gamma(\lambda+v)}{\Gamma(\lambda)}= \begin{cases}1, & (v=0 ; \lambda \in \mathbb{C} \backslash\{0\}), \\ \lambda(\lambda+1) \cdots(\lambda+k-1), & (v=k \in \mathbb{N} ; \lambda \in \mathbb{C}),\end{cases}
$$

is being understood conventionally that $(0)_{0}:=1$ and assumed tacitly that the $\Gamma$-quotient exists. For a widely-investigated $\lambda$-generalized version of the definition (2.13), see the work by Srivastava [22]. More interestingly, if we set $p \mapsto p+1 \quad\left(\rho_{1}=\cdots=\rho_{p}=1 ; \quad \lambda_{p+1}=\rho_{p+1}=1\right)$ and $q \mapsto q+1 \quad\left(\sigma_{1}=\cdots=\sigma_{q}=\right.$ $1 ; \quad \mu_{\mathrm{q}+1}=\beta ; \quad \sigma_{\mathrm{q}+1}=\alpha$ ), then the definition (2.13) (with $s=0$ ) reduces to the following generalized $M$-series which was introduced and studied recently (see [16, 18, 20]):

$$
\begin{aligned}
{ }_{p} M_{q}^{\alpha, \beta}\left(a_{1}, \cdots, a_{p} ; b_{1}, \cdots, b_{q} ; z\right) & =\sum_{k=0}^{\infty} \frac{\left(a_{1}\right)_{k} \cdots\left(a_{p}\right)_{k}}{\left(b_{1}\right)_{k} \cdots\left(b_{q}\right)_{k}} \frac{z^{k}}{\Gamma(\alpha k+\beta)} \\
& =\frac{1}{\Gamma(\beta)} p+1 \Psi_{q+1}^{*}\left[\begin{array}{c}
\left(a_{1}, 1\right), \cdots,\left(a_{p}, 1\right),(1,1) ; \\
\left(b_{1}, 1\right), \cdots,\left(b_{q}, 1\right),(\beta, \alpha) ;
\end{array}\right],
\end{aligned}
$$

or, equivalently,

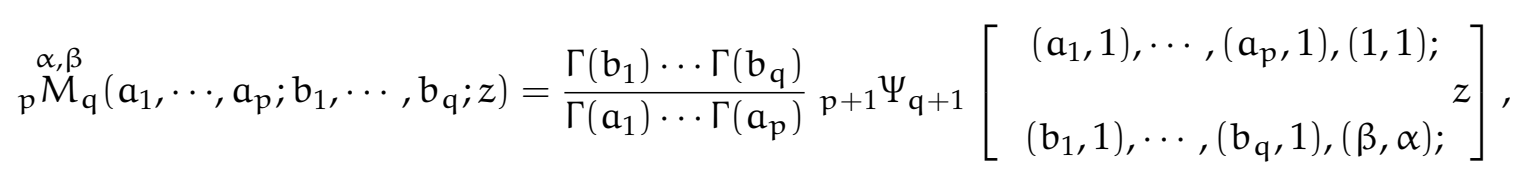

which reiterates the earlier authors' observation in (for example) [24] that the so-called generalized Mseries is, in fact, an obvious variant and special case of the Fox-Wright function ${ }_{p} \Psi_{q}$ or $p \Psi_{q}^{*}$ defined by (2.11).

\section{First set of results}

We begin by the following Definition 3.1 below.

Definition 3.1. For $\mathfrak{R}(\alpha)>0, \beta>0$ and $\mathfrak{R}(z)>0$, we define the functions $\mathcal{E}_{\alpha, n}, \mathcal{E}_{n, \beta}$ and $\varepsilon_{n}$ by

$$
\mathcal{E}_{\alpha, \mathrm{n}}(z):=z^{1-\mathrm{n}} \widetilde{\mathrm{E}}_{\alpha, \mathrm{n}}(z)= \begin{cases}z, & (\mathrm{n}=0), \\ \Gamma(\mathrm{n}) z \mathrm{E}_{\alpha, \mathrm{n}}(z)=z+\sum_{k=1}^{\infty} \frac{\Gamma(\mathrm{n})}{\Gamma(\alpha \mathrm{k}+\mathrm{n})} z^{k+1}, & (\mathrm{n} \in \mathbb{N}),\end{cases}
$$




$$
\mathcal{E}_{n, \beta}(z):=z^{1-\mathrm{n}} \widetilde{\mathrm{E}}_{n, \beta}(z)= \begin{cases}\frac{z}{1-z}, & (\mathrm{n}=0), \\ \Gamma(\beta) z \mathrm{E}_{n, \beta}(z)=z+\sum_{k=1}^{\infty} \frac{\Gamma(\beta)}{\Gamma(n k+\beta)} z^{k+1}, & (\mathrm{n} \in \mathbb{N}),\end{cases}
$$

and

$$
\varepsilon_{n}(z):=z^{1-n} \widetilde{\mathrm{E}}_{n}(z)= \begin{cases}\frac{z}{1-z}, & (\mathrm{n}=0), \\ z \mathrm{E}_{\mathrm{n}}(z)=z+\sum_{k=1}^{\infty} \frac{1}{\Gamma(n k+1)} z^{k+1}, & (\mathrm{n} \in \mathbb{N}),\end{cases}
$$

where the functions $\widetilde{\mathrm{E}}_{\alpha, n}(z), \widetilde{\mathrm{E}}_{n, \beta}(z)$, and $\widetilde{\mathrm{E}}_{n}(z)$ are given (as before) by (2.5), (2.6), and (2.7), respectively. Definition 3.2. In terms of Hadamard product (or convolution) given by (2.2), we define the following convolution operator:

$$
\mathfrak{F}_{\alpha, \beta}: \mathcal{A} \rightarrow \mathcal{A}
$$

by

$$
\mathfrak{F}_{\alpha, \beta}(\mathrm{f})(z):=\mathcal{E}_{\alpha, \beta}(z) * f(z)=z+\sum_{k=1}^{\infty} \frac{\Gamma(\beta)}{\Gamma(\alpha \mathrm{k}+\beta)} \mathrm{a}_{\mathrm{k}+1} z^{\mathrm{k}+1},
$$

where $\mathcal{E}_{\alpha, \beta}(z)$ is a unified version of the definitions (3.1), (3.2), and (3.3), which is given by

$$
\mathcal{E}_{\alpha, \beta}(z)=z+\sum_{k=1}^{\infty} \frac{\Gamma(\beta)}{\Gamma(\alpha \mathrm{k}+\beta)} z^{\mathrm{k}+1}, \quad(\min \{\mathfrak{R}(\alpha), \mathfrak{R}(\beta)\}>0 ; z \in \mathbb{U}) .
$$

The following identity follows readily from Definition 3.2:

$$
z\left(\mathfrak{F}_{\alpha, \beta+1}(f)(z)\right)^{\prime}=\left(\frac{\beta}{\alpha}\right) \mathfrak{F}_{\alpha, \beta}(f)(z)-\left(\frac{\beta-\alpha}{\alpha}\right) \mathfrak{F}_{\alpha, \beta+1}(f)(z), \quad(\mathfrak{R}(\alpha)>0 ; \mathfrak{R}(\beta)>-1 ; z \in \mathbb{U}) .
$$

Furthermore, we have

$$
\begin{aligned}
& \mathfrak{F}_{0, \beta}(f)(z)=f(z)=z+\sum_{k=2}^{\infty} a_{k} z^{k} \\
& \mathfrak{F}_{n, 1}(f)(z)=z+\sum_{k=1}^{\infty} \frac{1}{\Gamma(n k+1)} a_{k+1} z^{k+1}=\mathcal{E}_{n}(z) * f(z), \\
& \mathfrak{F}_{\alpha, n}(f)(z)=z+\sum_{k=1}^{\infty} \frac{\Gamma(n)}{\Gamma(\alpha k+n)} a_{k+1} z^{k+1}=\mathcal{E}_{\alpha, n}(z) * f(z),
\end{aligned}
$$

and

$$
\mathfrak{F}_{\mathfrak{n}, \beta}(\mathrm{f})(z)=z+\sum_{\mathrm{k}=1}^{\infty} \frac{\Gamma(\beta)}{\Gamma(\mathrm{nk}+\beta)} \mathrm{a}_{\mathrm{k}+1} z^{\mathrm{k}+1}=\mathcal{E}_{\mathrm{n}, \beta}(z) * \mathrm{f}(z) .
$$

By applying Theorem 2.1, we now prove the following results which provides an upper bound for the operator $\mathfrak{F}_{\alpha, \beta}$ defined by (3.4). For uniformity and convenience, we make use of the Fox-Wright ${ }_{p} \Psi_{q}$-notation in our results.

Theorem 3.3. Let $f(z) \in \mathcal{S}$. Then

$$
\left|\mathfrak{F}_{\alpha, \beta}(f)(z)\right| \leqq r \Gamma(\beta){ }_{1} \Psi_{1}\left[\begin{array}{c}
(2,1) ; \\
r \\
(\beta, \alpha) ;
\end{array}\right], \quad(|z| \leqq r(0<r<1) ; \alpha, \beta>0) .
$$


Proof. By hypothesis, the function $f(z) \in \mathcal{S}$ is of the form (2.1).

$$
\begin{aligned}
\left|\mathfrak{F}_{\alpha, \beta}(f)(z)\right| & \leqq r \sum_{k=0}^{\infty} \frac{(k+1) \Gamma(\beta)}{\Gamma(\alpha k+\beta)} \mathrm{r}^{k} \\
& =\mathrm{r} \Gamma(\beta) \sum_{k=0}^{\infty} \frac{\Gamma(k+2)}{k !} \frac{r^{k}}{\Gamma(\alpha k+\beta)}, \quad(|z| \leqq r \quad(0<r<1) ; \alpha, \beta>0),
\end{aligned}
$$

where we have used the following well-known estimate (see Theorem 2.1):

$$
\left|a_{k}\right| \leqq k \quad(k \in \mathbb{N} \backslash\{1\}) .
$$

The assertion (3.5) would follow from (3.6) when we interpret the series in (3.6) by means of the definition (2.1). Our demonstration of Theorem 3.3 is thus completed.

The proof of Theorem 3.4 below is analogous to that of Theorem 3.3.

Theorem 3.4. Let $\mathrm{f}(z) \in \mathcal{K}$. Then

$$
\left|\mathfrak{F}_{\alpha, \beta}(f)(z)\right| \leqq r \Gamma(\beta){ }_{1} \Psi_{1}\left[\begin{array}{c}
(1,1) ; \\
r \\
(\beta, \alpha) ;
\end{array}\right],(|z| \leqq r(0<r<1) ; \alpha, \beta>0) .
$$

We next prove the following result.

Theorem 3.5. If $\mathrm{f}(z) \in \mathcal{S}$, then

$$
\left|\mathfrak{F}_{\alpha, \beta}(f)(z)-z\right| \leqq \frac{\Gamma(\beta)}{\Gamma(\alpha+\beta)} \frac{r}{(1-r)^{2}}, \quad(|z| \leqq r(0<r<1) ; \alpha, \beta>0) .
$$

Proof. By supposing that the $f \in \mathcal{S}$ is given by (2.1), we have

$$
\left|\mathfrak{F}_{\alpha, \beta}(f)(z)-z\right|=\left|\sum_{k=2}^{\infty} \frac{\Gamma(\beta)}{\Gamma(\alpha k+\beta-\alpha)} a_{k} z^{k}\right|=\left|\sum_{k=2}^{\infty} \Omega(k) a_{k} z^{k}\right|,
$$

where

$$
\Omega(k)=\frac{\Gamma(\beta)}{\Gamma(\alpha k+\beta-\alpha)} \quad(k \in \mathbb{N} \backslash\{1\})
$$

Since, clearly,

$$
\Omega(k) \leqq \Omega(2)=\frac{\Gamma(\beta)}{\Gamma(\alpha+\beta)} \quad(k \in \mathbb{N} \backslash\{1\} ; \alpha, \beta>0)
$$

we conclude that

$$
\left|\mathfrak{F}_{\alpha, \beta}(f)(z)-z\right| \leqq \frac{\Gamma(\beta)}{\Gamma(\alpha+\beta)} \sum_{k=2}^{\infty}\left|a_{k}\right| \cdot|z|^{k} \leqq \frac{\Gamma(\beta)}{\Gamma(\alpha+\beta)}|z|^{2} \sum_{k=2}^{\infty} k r^{k-2} \leqq \frac{\Gamma(\beta)}{\Gamma(\alpha+\beta)} \frac{r}{(1-r)^{2}},
$$

where we have used the following estimates (see Theorem 2.1):

$$
|z|^{2}<1 \quad \text { and } \quad\left|a_{k}\right| \leqq k \quad(z \in \mathbb{U} ; k \in \mathbb{N} \backslash\{1\}) .
$$

This evidently completes the proof of Theorem 3.5. 


\section{Boundedness properties of the convolution operator $\mathfrak{F}_{\alpha, \beta}(f)$}

In this section, we investigate the boundedness properties of the convolution operator $\mathfrak{F}_{\alpha, \beta}(f)$ under mappings from a weighted $\mu$-Bloch space $B_{w}^{\mu}$ into a weighted-log Bloch space $B_{w, l o g}^{\mu}$. For all analytic functions $f \in \mathbb{U}$, the weighted $\mu$-Bloch space $B_{w}^{\mu}$ is defined by (see $[4,9]$ )

$$
\|f\|_{B_{w}^{\mu}}=\sup _{z \in \mathbb{U}}\left\{\left|f^{\prime}(z)\right| \frac{(1-|z|)^{\mu}}{w(1-|z|)}\right\}<\infty \quad(0<\mu<\infty)
$$

for $w:(0,1] \rightarrow[0, \infty)$. For a function $\varphi \in \mathrm{B}_{w}^{\mu}$, it is easy to see that

$$
\sup _{z \in \mathbb{U}}\left\{|\varphi(z)| \frac{(1-|z|)^{\mu}}{w(1-|z|)}\right\} \leqq c<\infty \quad(c>0 ; 0<\mu<\infty) .
$$

Moreover, the weighted logarithmic Bloch space $B_{w, l o g}^{\mu}$ of analytic functions $f(z)$ in $\mathbb{U}$ is defined by

$$
\|f\|_{B_{w, \log }^{\mu}}=\sup _{z \in \mathbb{U}}\left\{\left|f^{\prime}(z)\right| \frac{(1-|z|)^{\mu}}{w(1-|z|)} \log \left(\frac{1}{(1-|z|)}\right)\right\} \leqq c<\infty \quad(0<\mu<\infty)
$$

for $w:(0,1] \rightarrow[0, \infty)$. The weighted-log Bloch space $\mathrm{B}_{w, \log }^{\mu}$ is Banach space.

Our demonstration of Theorem 4.2 below would make use of the following lemma.

Lemma 4.1 ([15]). Let $\mathrm{f}$ and $\mathrm{g}$ be two analytic functions. Then

$$
z(g * f)^{\prime}(z):=g(z) * z f^{\prime}(z) \Longleftrightarrow(g * f)^{\prime}(z)=\frac{g(z)}{z} * f^{\prime}(z),
$$

provided that each member of (4.1) exists.

Theorem 4.2. Let $0<\mu<\infty, \alpha, \beta>0$, and $w:(0,1] \rightarrow[0, \infty)$, and let $f \in \mathcal{A}$ in the open unit disk $\mathbb{U}$. Then

$$
f \in B_{w}^{\mu} \Longleftrightarrow \mathfrak{F}_{\alpha, \beta}(f) \in B_{w}^{\mu} .
$$

Proof. By supposing $f \in B_{w}^{\mu}$, we find by Definition 3.2 and Lemma 4.1 on the weighted $\mu$-Bloch space that

$$
\left\|\mathfrak{F}_{\alpha, \beta}(\mathfrak{f})\right\|_{B_{w}^{\mu}}=\sup _{z \in \mathbb{U}}\left\{\left|\left(\mathcal{E}_{\alpha, \beta}(z) * f(z)\right)^{\prime}\right| \frac{(1-|z|)^{\mu}}{w(1-|z|)}\right\}=\sup _{z \in \mathbb{U}}\left\{\left|\frac{\mathcal{E}_{\alpha, \beta}(z)}{z} * f^{\prime}(z)\right| \frac{(1-|z|)^{\mu}}{w(1-|z|)}\right\} \leqq c|| f \|_{B_{w}^{\mu}}<\infty,
$$

where

$$
\sup _{z \in \mathbb{U}}\left\{\left|\mathcal{E}_{\alpha, \beta}(z)\right| \frac{(1-|z|)^{\mu}}{w(1-|z|)}\right\} \leqq c|z| \leqq c \quad(|z|<1 ; c>0)
$$

Consequently, we get

$$
\mathfrak{F}_{\alpha, \beta}(\mathrm{f}) \in \mathrm{B}_{w}^{\mu},
$$

which proves the first part (necessity) of Theorem 4.2.

Conversely, let us assume that $\mathfrak{F}_{\alpha, \beta}(f) \in B_{w}^{\mu}$. We then aim to show that

$$
\|\mathbf{f}\|_{B_{w}^{\mu}}=\sup _{z \in \mathbb{U}}\left\{\left|f^{\prime}(z)\right| \frac{(1-|z|)^{\mu}}{w(1-|z|)}\right\}<\infty \quad(0<\mu<\infty) .
$$

We now define an analytic function $\digamma \alpha, \beta(z)$ by

$$
\digamma_{\alpha, \beta}(z):=\frac{\mathcal{E}_{\alpha, \beta}(z)}{z} \quad(z \in \mathbb{U} \backslash\{0\}),
$$


such that

$$
\digamma_{\alpha, \beta}(z) * \digamma_{\alpha, \beta}^{(-1)}(z)=\frac{1}{1-z}
$$

Then, we find that

$$
\begin{aligned}
\|f\|_{B_{w}^{\mu}}=\sup _{z \in \mathbb{U}}\left\{\left|f^{\prime}(z)\right| \frac{(1-|z|)^{\mu}}{w(1-|z|)}\right\} & =\sup _{z \in \mathbb{U}}\left\{\left|\digamma_{\alpha, \beta}(z) * f^{\prime}(z) * \digamma_{\alpha, \beta}^{(-1)}(z)\right| \frac{(1-|z|)^{\mu}}{w(1-|z|)}\right\} \\
& =\sup _{z \in \mathbb{U}}\left\{\left|\left(\mathcal{E}_{\alpha, \beta}(z) * f^{\prime}(z)\right) * \digamma_{\alpha, \beta}^{(-1)}(z)\right| \frac{(1-|z|)^{\mu}}{w(1-|z|)}\right\} \leqq c_{\alpha, \beta}\left\|\mathfrak{F}_{\alpha, \beta}(f)\right\|_{B_{w}^{\mu}},
\end{aligned}
$$

where

$$
c_{\alpha, \beta}:=\left\|\digamma_{\alpha, \beta}^{(-1)}(z)\right\|
$$

This completes the proof of Theorem 4.2.

Theorem 4.3. Let $0<\mu<\infty, \alpha, \beta>0$, and $w:(0,1] \rightarrow[0, \infty)$. Also let $f \in \mathcal{A}$ in the open unit disk $\mathbb{U}$. Then

$$
\left\|\mathfrak{F}_{\alpha, n}(f)\right\|_{B_{w}^{\mu}} \leqslant c \mid f \|_{B_{w, \log }^{\mu}} \quad(c>0) .
$$

Proof. For a function $\varphi \in \mathrm{B}_{w}^{\mu}$, we see that, for every $\varepsilon>0$, there is an $\mathfrak{r}(0<\mathfrak{r}<1)$ such that

$$
|\varphi(z)| \leqq \varepsilon \log \left(\frac{1}{1-|z|}\right) \quad(\mathfrak{r}<|z|<1) .
$$

Therefore, $w:(0,1] \rightarrow[0, \infty)$, we have

$$
\begin{aligned}
\left\|\mathfrak{F}_{\alpha, \beta}(f)\right\|_{B_{w}^{\mu}} & =\sup _{z \in \mathbb{U}}\left\{\left|\left(\mathcal{E}_{\alpha, \beta}(z) * f(z)\right)^{\prime}\right| \frac{(1-|z|)^{\mu}}{w(1-|z|)}\right\} \\
& \leqq \varepsilon \sup _{z \in \mathbb{U}}\left\{\left|f^{\prime}(z)\right| \frac{(1-|z|)^{\mu}}{w(1-|z|)} \log \left(\frac{1}{1-|z|}\right)\right\} \quad(\mathfrak{r}<|z|<1) \\
& \leqq \varepsilon \varepsilon f \|_{B_{w, \log }^{\mu}}<\infty,
\end{aligned}
$$

where

$$
\left|\mathcal{E}_{\alpha, \beta}(z)\right| \leqq \varepsilon|z| \log \left(\frac{1}{1-|z|}\right) \leqq \varepsilon \log \left(\frac{1}{1-|z|}\right) \quad(|z|<1) .
$$

The proof of Theorem 4.3 is thus completed.

Theorem 4.4. Let $0<\mu<\infty, \alpha, \beta>0$, and $w:(0,1] \rightarrow[0, \infty)$. Then

$$
\left\|\mathfrak{F}_{\alpha, \beta}(f)\right\|_{B_{w}^{\mu}} \leqq \frac{\varepsilon \Gamma(\beta)}{2}\|f(z)\|_{B_{w, \log }^{\mu}} \quad(\varepsilon>0) .
$$

Proof. Just as in (4.2), suppose that, for a function $\varphi \in B_{w}^{\mu}$ and for every $\varepsilon>0$, there is an $\mathfrak{r}(0<\mathfrak{r}<1)$ such that

$$
|\varphi(z)| \leqq \frac{\varepsilon}{2} \log \left(\frac{1}{1-|z|}\right) \quad(\mathfrak{r}<|z|<1) .
$$

Therefore, for $w:(0,1] \rightarrow[0, \infty)$, we find that

$$
\left\|\mathfrak{F}_{\alpha, \beta}(\mathbf{f})\right\|_{B_{w}^{\mu}} \leqq \Gamma(\beta) \sup _{z \in \mathbb{U}}\left\{\left|\left(\mathfrak{r}\left({ }_{1} \Psi_{1}\left[\begin{array}{c}
(1,1) ; \\
(\beta, \alpha) ;
\end{array}\right] * \mathfrak{r}(\mathfrak{r})\right)\right)^{\prime}\right| \frac{(1-|z|)^{\mu}}{w(1-|z|)}\right\}
$$




$$
\begin{aligned}
& =\Gamma(\beta) \sup _{z \in \mathbb{U}}\left\{\left|\left({ }_{1} \Psi_{1}\left[\begin{array}{c}
(1,1) ; \\
(\beta, \alpha) ;
\end{array}\right] * f(\mathfrak{r})\right)+\mathfrak{r}\left({ }_{1} \Psi_{1}\left[\begin{array}{r}
(1,1) ; \\
(\beta, \alpha) ;
\end{array}\right] * \mathfrak{r}(\mathfrak{r})\right)^{\prime}\right| \frac{(1-|z|)^{\mu}}{w(1-|z|)}\right\} \\
& =\Gamma(\beta) \sup _{z \in \mathbb{U}}\left\{\left|{ }_{1} \Psi_{1}\left[\begin{array}{c}
(1,1) ; \\
(\beta, \alpha) ;
\end{array}\right] *\left[\mathbf{f}(\mathfrak{r})+\mathfrak{r} f^{\prime}(\mathfrak{r})\right]\right| \frac{(1-|z|)^{\mu}}{\mathcal{w}(1-|z|)}\right\} \\
& \leqq \varepsilon \Gamma(\beta) \sup _{z \in \mathbb{U}}\left\{\left|\frac{(\mathfrak{r} f(\mathfrak{r}))^{\prime}}{2}\right| \frac{(1-|z|)^{\mu}}{w(1-|z|)} \log \left(\frac{1}{1-|z|}\right)\right\} \\
& \leqq \frac{\varepsilon \Gamma(\beta)}{2}\|\mathfrak{f}(z)\|_{B_{w, \log }^{\mu} \quad(\mathfrak{r}<|z|<1 ; \varepsilon>0) .}
\end{aligned}
$$

The proof of Theorem 4.4 is thus completed.

\section{Conclusion}

Our present investigation was motivated essentially by the fact that studies of convolution play an important role in Geometric Function Theory (GFT). In recent years, such studies have attracted a large number of researchers who made use of the Hadamard product (or convolution) to introduce and investigate various interesting subclasses of analytic and univalent functions for such well-known concepts as the subordination and superordination inequalities, integral mean and partial sums, and so on. Here, in this article, we have applied the Hadamard product (or convolution) by utilizing some special functions. Our contribution in this paper includes defining a new linear operator in the form of the generalized Mittag-Leffler function in terms of the extensively-investigated Fox-Wright ${ }_{p} \Psi_{q}$-function in right-half of the open unit disk $\mathbb{U}$ where $\mathfrak{R}(z)>0$. We have then shown that the newly-defined linear convolution operator is bounded in some spaces. In particular, we have presented several boundedness properties of this linear convolution operator under mappings from a weighted Bloch space into a weighted-log Bloch space. For uniformity and convenience, we have chosen to make use of the Fox-Wright ${ }_{\mathrm{p}} \Psi_{\mathrm{q}}$-notation in our results.

\section{Acknowledgment}

The authors are grateful to the referees for delicately reading the paper and for their valuable comments.

\section{References}

[1] Z. E. Abdulnaby, R. W. Ibrahim, A. Kılıçman, Some properties for integro-differential operator defined by a fractional formal, Springer Plus., 5 (2016), 1-9. 1

[2] Z. E. Abdulnaby, H. M. Srivastava, A. Kılıçman, R. W. Ibrahim, A novel subclass of analytic functions specified by a family of fractional derivatives in the complex domain, Filomat, 31 (2017), 2837-2849. 1

[3] R. P. Agarwal, A propos d'une note de M, Pierre Humbert, C. R. Acad. Sci. Paris., 236 (1953), 2031-2032. 2.3

[4] P. L. Duren, Univalent Functions, Springer-Verlag, New York, (1983). 2.1, 4

[5] R. Gorenflo, A. A. Kilbas, F. Mainardi, V. R. Sergei, Mittag-Leffler Functions, Related Topics and Applications, Springer, Berlin, (2014). 1, 2

[6] H. J. Haubold, A. M. Mathai, R. K. Saxena, Mittag-Leffler functions and their applications, J. Appl. Math., 2011 (2011), 51 pages. 1,2

[7] P. Humbert, Quelques résultats relatifs à la fonction de Mittag-Leffler, C. R. Acad. Sci. Paris, 236 (1953), 1467-1468. 2.3

[8] A. A. Kilbas, M. Saigo, R. K. Saxena, Generalized Mittag-Leffler function and generalized fractional calculus operators, Integral Transforms Spec. Funct., 15 (2004), 31-49. 2

[9] A. A. Kilbas, H. M. Srivastava, J. J. Trujillo, Theory and Applications of Fractional Differential Equations, Elsevier Science B.V., Amsterdam, (2006). 2, 2, 4

[10] A. Kılıçman, R. W. Ibrahim, Z. E. Abdulnaby, On a generalized fractional integral operator in a complex domain, Appl. Math. Inf. Sci., 10 (2016), 1053-1059. 1 
[11] V. S. Kiryakova, Multiple (multiindex) Mittag-Leffler functions and relations to generalized fractional calculus, J. Comput. appl. Math., 118 (2000), 241-259. 2

[12] Y. Li, Y. Q. Chen, I. Podlubny, Mittag-Leffler stability of fractional order nonlinear dynamic systems, Automatica J. IFAC, 45 (2009), 1965-1969. 2

[13] G. M. Mittag-Leffler, Sur la nouvelle fonction $\mathrm{E}_{\alpha}(x)$, C. R. Acad. Sci. Paris, (Ser. II), 137 (1903), 554-558. 2.3

[14] J. Paneva-Konovska, Series in Mittag-Leffler functions: inequalities and convergent theorems, Fract. Calc. Appl. Anal., 13 (2010), 403-414. 2, 2, 2

[15] S. Ruscheweyh, Convolutions in Geometric Function Theory, Séminaire de Mathématiques (NATO Advanced Study Institute), Les Presses de 1'Universitéde Montréal, Montréal, Québec, (1982). 4.1

[16] R. K. Saxen, A remark on a paper on M-series, Fract. Calc. Appl. Anal., 12 (2009), 109-110. 2, 2

[17] R. K. Saxena, J. Ram, M. Vishnoi, Certain properties of generalized Mittag-Leffler function, In Proceedings of the 3rd Annual Conference of the Society for Special Functions and Their Applications, Soc. Spec. Funct. Appl., (2002), 75-81. 2

[18] M. Sharma, Fractional integration and fractional differentiation of the M-series, Fract. Calc. Appl. Anal., 11 (2008), 187-191. 2, 2

[19] K. Sharma, Application of fractional calculus operators to related areas, Gen. Math. Notes., 7 (2011), 33-40.

[20] M. Sharma, R. Jain, A note on a generalized M-series as a special function of fractional calculus, Fract. Calc. Appl. Anal., 12 (2009), 449-452. 2, 2

[21] H. M. Srivastava, On an extension of the Mittag-Leffler function, Yokohama Math. J., 16 (1968), 77-88. 2

[22] H. M. Srivastava, A new family of the $\lambda$-generalized Hurwitz-Lerch zeta functions with application, Appl. Math. Inform. Sci., 8 (2014), 1485-1500. 2

[23] H. M. Srivastava, R. K. Saxena, Operators of fractional integration and their applications, Appl. Math. Comput., 118 (2001), 1-52. 2

[24] H. M. Srivastava, R. K. Saxena, T. K. Pogány, R. Saxena, Integral and computational representations of the extended Hurwitz-Lerch zeta function, Integral Transforms Spec. Funct., 22 (2011), 487-506. 2, 2, 2

[25] H. M. Srivastava, R. K. Saxena, C. Ram, A unified presentation of the Gamma-type functions occurring in diffraction theory and associated probability distributions, Appl. Math. comput., 162 (2005), 931-947. 2

[26] H. M. Srivastava, Ž. Tomovski, Fractional calculus with an integral operator containing a generalized MittagLeffler function in the kernel, J. Appl. Math. Comput., 211 (2009), 198-210 1, 2

[27] Ž. Tomovski, R. Hilfer, H. M. Srivastava, Fractional and operational calculus with generalized fractional derivative operators and Mittag-Leffler type functions, Integral Transforms and Spec. Funct., 21 (2010), 797-814. 1, 2

[28] A. Wiman, Über den Fundamentalsatz in der Teorie der Funktionen $\mathrm{E}_{\alpha}$ (x), Acta. Math., 29 (1905), 191-201. 2.3 